PROCEEDINGS OF THE

AMERICAN MATHEMATICAL SOCIETY

Volume 125, Number 10, October 1997, Pages 2953-2961

S 0002-9939(97)03969-5

\title{
ELLIPTIC EIGENVALUE PROBLEMS WITH HIGHLY DISCONTINUOUS NONLINEARITIES
}

\author{
SALVATORE A. MARANO
}

(Communicated by Palle E. T. Jorgensen)

\begin{abstract}
For a family of elliptic eigenvalue problems with highly discontinuous nonlinearities, the existence of unbounded continua of positive solutions containing $(0,0)$ is established by using techniques and results from set-valued analysis. Some special cases are then presented and discussed.
\end{abstract}

\section{INTRODUCTION}

The structure of the solution set to large classes of nonlinear elliptic eigenvalue problems has been widely investigated, both in general settings [2], [5], [7], [13], [17], [18], [23], [24] and related to applications [3], [7], [13], [14]. Concerning this field of research, several specific models arising from mathematical physics naturally lead to considering elliptic equations with nonlinear terms discontinuous at a finite or countable set of points. In such a case, somewhat complete and satisfactory results are already available; see for instance [3], [5], [7], [13], [14], [17], the very recent monograph [11], and the references given there. Hence, from a theoretical point of view, it is meaningful to ask whether analogous properties also hold for elliptic eigenvalue problems having much more discontinuous nonlinearities.

The main purpose of the present paper is to provide a contribution in the abovementioned direction. Accordingly, here, we study the Dirichlet boundary-value problem for semilinear elliptic equations of the type

$$
-\sum_{i, j=1}^{n} a_{i j}(x, u) \frac{\partial^{2} u}{\partial x_{i} \partial x_{j}}=f(x, u, \lambda) \quad \text { in } \Omega,
$$

where $\Omega$ denotes a bounded domain in $\mathbb{R}^{n}(n \geq 3)$ with a smooth boundary, the coefficients $a_{i j}(x, z)$ satisfy Carathéodory's conditions and belong to a family of functions (previously introduced in [19]) having vanishing mean oscillation with respect to $x$ locally uniformly in $z$, while the nonlinear term $f: \Omega \times \mathbb{R}_{0}^{+} \times \mathbb{R}_{0}^{+} \rightarrow \mathbb{R}_{0}^{+}$ is discontinuous with respect to the second variable at a set of points of Lebesgue measure zero only. Since both the number $\lambda$ and the function $u$ are unknown quantities in (E), we define a positive solution of $(\mathrm{E})$ to be an ordered pair $(\lambda, u)$

Received by the editors March 15, 1996 and, in revised form, May 7, 1996.

1991 Mathematics Subject Classification. Primary 35J65, 35B30, 35R70.

Key words and phrases. Elliptic eigenvalue problems, discontinuous nonlinearities, elliptic differential inclusions, unbounded continuum of solutions.

Work performed under the auspices of G.N.A.F.A. of C.N.R. and partially supported by M.U.R.S.T. of Italy $(40 \%, 1994)$.

(C) 1997 American Mathematical Society 
such that $\lambda \in \mathbb{R}_{0}^{+}, u \in W^{2, p}(\Omega) \cap W_{0}^{1, p}(\Omega)$ for some $\left.p \in\right] n,+\infty[, u(x) \geq 0$ in $\Omega$, and $-\sum_{i, j=1}^{n} a_{i j}(x, u(x)) \frac{\partial^{2} u(x)}{\partial x_{i} \partial x_{j}}=f(x, u(x), \lambda)$ for almost every $x \in \Omega$.

As in our previous articles [6], [15], [16], the approach we develop uses techniques and results from set-valued analysis. We first consider an appropriate upper semicontinuous convex-valued regularization $F(x, z, \lambda)$ of $f(x, z, \lambda)$ and, by means of fixed point arguments mainly based upon [7, Theorem 2.12], we prove that there exists an unbounded continuum of positive solutions to the elliptic differential inclusion

$$
-\sum_{i, j=1}^{n} a_{i j}(x, u) \frac{\partial^{2} u}{\partial x_{i} \partial x_{j}} \in F(x, u(x), \lambda) \quad \text { in } \Omega,
$$

which contains $(0,0)$; see Theorem 2.1. Next, through a technical lemma (Proposition 1.2) and a suitable assumption, we achieve the same conclusion for (E). The result thus established (Theorem 2.3) can be regarded as a highly discontinuous version of [23, Theorem 2.12] and [24, Theorem 3.7]. It improves in several directions Theorem 4.4 of [17] and Theorem 3.3 in [18], and, concerning the nonlinearity $f$, exhibits more general hypotheses than Theorem 19 of [13] as well as Theorem 5.2 in [7]. Further, when applied to less abstract situations, it takes simpler and practical forms; see for example Theorems 2.4 and 2.5.

This paper is organized in three sections, including the Introduction. Notations, basic definitions, and preliminary results are collected in Section 1. The main theorems together with some special cases, interesting enough to be explicitly considered, are presented and discussed in Section 2.

\section{BASIC DEFINITIONS AND PRELIMINARY RESULTS}

A set in a topological space $X$ is said to be a subcontinuum of $X$ provided it is nonempty, closed, and connected.

Let $(Z,\|\cdot\|)$ be a real Banach space and let $W$ be a nonempty subset of $Z$. We write $\bar{W}$ for the closure of $W, c o(W)$ for the convex hull of $W,|W|$ for $\sup _{w \in W}\|w\|$. Denote by $\theta$ the zero vector of $Z$. A cone in $Z$ is a nonempty, convex, closed set $K \subseteq Z$ satisfying

$$
K \cap(-K)=\{\theta\} \quad \text { and } \quad \alpha K \subseteq K \quad \text { for every } \quad \alpha \in \mathbb{R}_{0}^{+} .
$$

If $K$ indicates a cone of $Z$, then the pair $(Z, K)$ is usually called an ordered Banach space.

Let $X$ be a nonempty set. The symbols $\Phi: X \rightarrow 2^{Z}$ mean that $\Phi$ is a multifunction from $X$ into $Z$, namely a function which assigns to each point $x \in X$ a nonempty closed subset $\Phi(x)$ of $Z$. The graph of $\Phi$ is the set $\{(x, z) \in X \times Z$ : $z \in \Phi(x)\}$. For $W \subseteq Z$, define $\Phi^{-}(W)=\{x \in X: \Phi(x) \cap W \neq \emptyset\}$. If $(X, \mathcal{F})$ is a measurable space and $\Phi^{-}(W) \in \mathcal{F}$ for every open set $W \subseteq Z$, we say that $\Phi$ is measurable. When $X$ is a topological space and the set $\Phi^{-}(W)$ is closed for every closed subset $W$ of $Z$, the multifunction $\Phi$ is called upper semicontinuous. In such a case the graph of $\Phi$ is clearly closed in $X \times Z$. Conversely, if $\Phi$ has compact values, closed graph, and to each $x \in X$ there corresponds a neighbourhood $V$ of $x$ so that $\overline{\Phi(V)}$ is compact, then it is also upper semicontinuous (see for instance [4, Lemma 2.9]). The multifunction $\Phi$ is said to be completely continuous provided it 
has convex values, is upper semicontinuous, and maps bounded subsets of $X$ into relatively compact subsets of $Z$.

By using Theorem 2.12 of [7] it is a simple matter to deduce the following result, which can be regarded as a multivalued version of [2, Theorem 17.1].

Theorem 1.1. Let $\Psi: \mathbb{R}_{0}^{+} \times K \rightarrow 2^{K}$ be a completely continuous multifunction and let $\Sigma=\left\{(\lambda, z) \in \mathbb{R}_{0}^{+} \times K: z \in \Psi(\lambda, z)\right\}$. Suppose $\Psi(0, \theta)=\{\theta\}, z \in \Psi(0, z)$ implies $z=\theta$, and there exists $\rho>0$ such that $\alpha z \notin \Psi(0, z)$ for every $\alpha \geq 1$ and every $z \in K$ with $\|z\|=\rho$. Then the set $\Sigma$ contains an unbounded subcontinuum emanating from $(0, \theta)$.

Throughout this paper the symbol $\Omega$ denotes a nonempty, bounded, open, and connected subset of $\mathbb{R}^{n}, n \geq 3$, with a boundary of class $C^{1,1}$, while $p$ is any real number greater than $n$. Moreover, from now on, 'measurable' simply means Lebesgue measurable and $m(E)$ indicates the measure of $E$. Given a nonnegative integer $k$, we denote by $W^{k, p}(\Omega)$ the space of all real-valued functions defined on $\Omega$ whose weak partial derivatives up to the order $k$ lie in $L^{p}(\Omega)$, equipped with the usual norm $\|\cdot\|_{k, p}$. The symbol $W_{0}^{1, p}(\Omega)$ stands for the closure of $C_{0}^{\infty}(\Omega)$ in $W^{1, p}(\Omega)$. Finally, to shorten notations, we set

$$
X_{p}(\Omega)=W^{2, p}(\Omega) \cap W_{0}^{1, p}(\Omega) .
$$

In $\mathbb{R}_{0}^{+} \times X_{p}(\Omega)$ we will always consider the cartesian product topology.

Let $L$ be the second order, semilinear elliptic differential operator defined by

$$
L u=-\sum_{i, j=1}^{n} a_{i j}(x, u) \frac{\partial^{2} u}{\partial x_{i} \partial x_{j}},
$$

where the functions $a_{i j}: \Omega \times \mathbb{R} \rightarrow \mathbb{R}, i, j=1,2, \ldots, n$, satisfy the following hypotheses [19, p. 2483].

$\left(i_{1}\right)$ Carathéodory's conditions: $x \rightarrow a_{i j}(x, z)$ is measurable for each $z \in \mathbb{R}$ and $z \rightarrow a_{i j}(x, z)$ is continuous for almost all $x \in \Omega$.

$\left(i_{2}\right)$ Uniform ellipticity: $a_{i j}=a_{j i}$ and there exists $\alpha>0$ such that

$$
\sum_{i, j=1}^{n} a_{i j}(x, z) \xi_{i} \xi_{j} \geq \alpha \sum_{i=1}^{n} \xi_{i}^{2}
$$

almost everywhere in $\Omega$, for every $z \in \mathbb{R},\left(\xi_{1}, \xi_{2}, \ldots, \xi_{n}\right) \in \mathbb{R}^{n}$.

$\left(i_{3}\right)$ Local uniform continuity with respect to $z$, uniformly in $x: a_{i j}(\cdot, 0) \in L^{\infty}(\Omega)$; further, there are $a \in L^{\infty}(\Omega)$ and, for each $r>0$, a nondecreasing function $\omega_{r}: \mathbb{R}_{0}^{+} \rightarrow \mathbb{R}_{0}^{+}$satisfying $\lim _{t \rightarrow 0^{+}} \omega_{r}(t)=0$ as well as

$$
\left|a_{i j}\left(x, z^{\prime}\right)-a_{i j}\left(x, z^{\prime \prime}\right)\right| \leq a(x) \omega_{r}\left(\left|z^{\prime}-z^{\prime \prime}\right|\right)
$$

almost everywhere in $\Omega$, for all $z^{\prime}, z^{\prime \prime} \in[-r, r]$.

$\left(i_{4}\right)$ Vanishing mean oscillation with respect to $x$, locally uniformly in $z$ : To every $r>0$ there corresponds a function $\eta_{r}: \mathbb{R}^{+} \rightarrow \mathbb{R}^{+}$such that

$$
\sup _{\rho \leq t} \frac{1}{m\left(\Omega_{\rho}\right)} \int_{\Omega_{\rho}}\left|a_{i j}(x, z)-\frac{1}{m\left(\Omega_{\rho}\right)} \int_{\Omega_{\rho}} a_{i j}\left(x^{\prime}, z\right) d x^{\prime}\right| d x \leq \eta_{r}(t)
$$

for all $z \in[-r, r]$ and $\lim _{t \rightarrow 0^{+}} \eta_{r}(t)=0$. Here $\Omega_{\rho}=\Omega \cap B_{\rho}$, where $B_{\rho}$ ranges in the class of balls with radius $\rho$ centered at the points of $\Omega$. 
Owing to Lemma 2.1 of [19] and Theorem 4.4 in [8], for each $w \in C^{1}(\bar{\Omega})$, the linear elliptic operator (induced by $L$ )

$$
L_{w} u=-\sum_{i, j=1}^{n} a_{i j}(x, w(x)) \frac{\partial^{2} u}{\partial x_{i} \partial x_{j}}
$$

is bijective between $X_{p}(\Omega)$ and $L^{p}(\Omega)$. Using the Rellich-Kondrachov theorem [1, Theorem 6.2] and arguing as in [19, p. 2491] (see also [20]), we infer the following

Proposition 1.1. Let $\Gamma: C^{1}(\bar{\Omega}) \times L^{p}(\Omega) \rightarrow C^{1}(\bar{\Omega})$ be the operator defined by $\Gamma(w, v)=u$ for every $(w, v) \in C^{1}(\bar{\Omega}) \times L^{p}(\Omega)$, where $u$ is the unique solution to the Dirichlet problem

$$
L_{w} u=v(x) \quad \text { almost everywhere in } \Omega, \quad u \in X_{p}(\Omega) .
$$

Then $\Gamma$ is continuous and maps bounded subsets of $C^{1}(\bar{\Omega}) \times L^{p}(\Omega)$ into relatively compact subsets of $C^{1}(\bar{\Omega})$.

We next point out that, by the maximum principle [10, Theorem 9.6], the assertions $u \in X_{p}(\Omega), L u(x) \geq 0$ for almost every $x \in \Omega$ immediately lead to $u(x) \geq 0$ in $\Omega$.

Finally, a simple argument based on Lemma 1 of [9] and Lemma 7.7 in [10] yields the following

Proposition 1.2. Let $u \in W^{2, p}(\Omega)$ and let $E$ be a measurable subset of $\mathbb{R}$ such that $m(E)=0$. Then $L u(x)=0$ for almost every $x \in u^{-1}(E)$.

\section{EXISTENCE OF UNBOUNDED CONTINUA OF SOLUTIONS}

This section is arranged into two parts. The first contains an existence result of unbounded continua of solutions to a class of elliptic differential inclusions depending on a parameter, while the second treats elliptic eigenvalue problems with highly discontinuous nonlinearities.

2.1. Elliptic differential inclusions. Let $F: \Omega \times \mathbb{R}_{0}^{+} \times \mathbb{R}_{0}^{+} \rightarrow 2^{\mathbb{R}_{0}^{+}}$be a convexvalued multifunction. We will make the assumptions:

$\left(a_{1}\right) x \rightarrow F(x, z, \lambda)$ is measurable for every $z, \lambda \in \mathbb{R}_{0}^{+}$.

$\left(a_{2}\right)(z, \lambda) \rightarrow F(x, z, \lambda)$ is upper semicontinuous for almost all $x \in \Omega$.

$\left(a_{3}\right)$ To each $r>0$ there corresponds a function $M_{r} \in L^{p}(\Omega)$ such that $\sup \{|F(x, z, \lambda)|: z, \lambda \in[0, r]\} \leq M_{r}(x)$ almost everywhere in $\Omega$.

$\left(a_{4}\right) F(x, 0,0)=\{0\}$ for almost all $x \in \Omega$.

$\left(a_{5}\right)$ The problem $L u \in \lambda F(x, u, 0)$ in $\Omega, u \in X_{p}(\Omega)$, has no nontrivial solutions for every $\lambda \in] 0,1]$.

Define

$\Sigma_{F}=\left\{(\lambda, u) \in \mathbb{R}_{0}^{+} \times X_{p}(\Omega): L u(x) \in F(x, u(x), \lambda)\right.$ almost everywhere in $\left.\Omega\right\}$,

where $L$ is given by (1). The following theorem will be used to prove the main result of the next part. It also seems interesting enough to be considered separately.

Theorem 2.1. Let $F: \Omega \times \mathbb{R}_{0}^{+} \times \mathbb{R}_{0}^{+} \rightarrow 2^{\mathbb{R}_{0}^{+}}$be a convex-valued multifunction fulfilling hypotheses $\left(a_{1}\right)-\left(a_{5}\right)$. Then there exists an unbounded subcontinuum of the set $\Sigma_{F}$ which contains $(0,0)$. 
Proof. Write $K=\left\{u \in C^{1}(\bar{\Omega}): u(x) \geq 0\right.$ in $\left.\bar{\Omega}\right\}$ and, for $(\lambda, u) \in \mathbb{R}_{0}^{+} \times K$,

$$
\Phi(\lambda, u)=\left\{v \in L^{p}(\Omega): v(x) \in F(x, u(x), \lambda) \text { almost everywhere in } \Omega\right\} .
$$

Because of $\left(a_{3}\right)$ and [4, Lemma 7.1] one has $\Phi(\lambda, u) \neq \emptyset$. Moreover, $\Phi(\lambda, u)$ is clearly convex and closed. The same arguments used in [22, Proposition 1.4] ensure that the multifunction $\Phi: \mathbb{R}_{0}^{+} \times K \rightarrow 2^{L^{p}(\Omega)}$ has the following properties:

(i) $\left(\lambda_{h}, u_{h}\right) \rightarrow(\lambda, u)$ in $\mathbb{R}_{0}^{+} \times K, v_{h} \rightarrow v$ weakly in $L^{p}(\Omega), v_{h} \in \Phi\left(\lambda_{h}, u_{h}\right)$ for all $h \in \mathbb{N}$ imply $v \in \Phi(\lambda, u)$.

(ii) $\Phi$ maps bounded subsets of $\mathbb{R}_{0}^{+} \times K$ into weakly relatively compact subsets of $L^{p}(\Omega)$.

We next define $\Psi(\lambda, u)=\Gamma(u, \Phi(\lambda, u)),(\lambda, u) \in \mathbb{R}_{0}^{+} \times K$, as well as

$$
\Sigma=\left\{(\lambda, u) \in \mathbb{R}_{0}^{+} \times K: u \in \Psi(\lambda, u)\right\},
$$

where $\Gamma$ denotes the operator introduced in Proposition 1.1. Since a straightforward computation yields $\Sigma \subseteq \Sigma_{F}$, the conclusion is achieved by proving that all the assumptions of Theorem 1.1 are fulfilled.

Obviously, $\Psi(\lambda, u)$ is nonempty and convex. Owing to the maximum principle (see for instance [10, Theorem 9.6]), the inclusion $\Psi(\lambda, u) \subseteq K$ holds. Proposition 1.1 combined with (ii) guarantees that $\Psi(\lambda, u)$ is also closed for all $(\lambda, u) \in \mathbb{R}_{0}^{+} \times K$ and the multifunction $\Psi: \mathbb{R}_{0}^{+} \times K \rightarrow 2^{K}$ maps bounded sets into relatively compact sets. Let us verify that $\Psi$ has a closed graph. If $\left(\lambda_{h}, u_{h}\right) \rightarrow(\lambda, u)$ in $\mathbb{R}_{0}^{+} \times K$, $w_{h} \rightarrow w$ in $K$, and $w_{h} \in \Psi\left(\lambda_{h}, u_{h}\right), h \in \mathbb{N}$, then to each $h \in \mathbb{N}$ there corresponds $v_{h} \in \Phi\left(\lambda_{h}, u_{h}\right)$ satisfying $w_{h}=\Gamma\left(\lambda_{h}, u_{h}\right)$. On account of (ii) again, the set $\bigcup_{h \in \mathbb{N}} \Phi\left(\lambda_{h}, u_{h}\right)$ is weakly relatively compact. Hence, by taking a subsequence if necessary, we may suppose $v_{h} \rightarrow v$ weakly in $L^{p}(\Omega)$. Property (i) leads to $v \in \Phi(\lambda, u)$, namely $w \in \Psi(\lambda, u)$. The preceding arguments ensure that the multifunction $\Psi$ is completely continuous. Moreover one has $\Psi(0,0)=\{0\}$ because, owing to $\left(a_{4}\right)$, $\Phi(0,0)=\{0\}$. Bearing in mind $\left(a_{5}\right)$ we easily infer $\alpha u \notin \Psi(0, u)$ whenever $\alpha \geq 1$ and $u \in K \backslash\{0\}$, so that all the hypotheses of Theorem 1.1 hold. This completes the proof.

Remark 2.1. Theorem 2.1 is actually true for any elliptic operator satisfying the maximum principle and the conclusion of Proposition 1.1. Besides $L$, the linear operators considered in [2], [3], [5], [6], [14], [17], [18] clearly are of the abovementioned kind.

Remark 2.2. Assumption $\left(a_{5}\right)$ of Theorem 2.1 may evidently be replaced by the less restrictive one:

$\left(a_{5}\right)^{\prime}$ There is $\rho>0$ such that the problem $L u \in \lambda F(x, u, 0)$ in $\Omega, \sup _{x \in \Omega} u(x)=\rho$, has no solutions belonging to $X_{p}(\Omega)$ for all $\left.\left.\lambda \in\right] 0,1\right]$.

This condition is rather implicit. However, in some concrete situations it becomes much easier to verify. For instance, when $-L=\Delta$ (the Laplace operator), $B$ denotes the same constant as [15, p. 170], and $B\left\|M_{\rho}\right\|_{p}<\rho$ for a suitable $\rho>0$, then $\left(a_{5}\right)^{\prime}$ is of course fulfilled.

Theorem 2.1 has a variety of simple and practical special cases. As an example we state the result below, which improves in several directions Theorem 3.1 of [17] and Theorem 2.1 in [18]. 
Theorem 2.2. Suppose $F$ complies with hypotheses $\left(a_{1}\right)-\left(a_{3}\right)$. Then the set $\{(\lambda, u)$ $\in \mathbb{R}_{0}^{+} \times X_{p}(\Omega): L u(x) \in \lambda F(x, u(x), \lambda)$ almost everywhere in $\left.\Omega\right\}$ contains an unbounded subcontinuum emanating from $(0,0)$.

2.2. Elliptic equations with discontinuous nonlinearities. Given a function $f: \Omega \times \mathbb{R}_{0}^{+} \times \mathbb{R}_{0}^{+} \rightarrow \mathbb{R}_{0}^{+}$, the conditions below will be posited in what follows.

$\left(h_{1}\right)$ There exists $\Omega_{0} \subseteq \Omega$ with $m\left(\Omega_{0}\right)=0$ such that the set

$$
D_{f}=\bigcup_{x \in \Omega \backslash \Omega_{0}} \bigcup_{\lambda \in \mathbb{R}_{0}^{+}}\left\{z \in \mathbb{R}_{0}^{+}: f(x, \cdot, \lambda) \text { is discontinuous at } z\right\}
$$

has measure zero.

$\left(h_{2}\right) x \rightarrow f(x, z, \lambda)$ is measurable for all $z \in \mathbb{R}_{0}^{+} \backslash D_{f}, \lambda \in \mathbb{R}_{0}^{+}$.

$\left(h_{3}\right) \lambda \rightarrow f(x, z, \lambda)$ is continuous for almost every $x \in \Omega$, locally uniformly in $z \in \mathbb{R}_{0}^{+} \backslash D_{f}$.

$\left(h_{4}\right)$ To each $r>0$ there corresponds a function $\mu_{r} \in L^{p}(\Omega)$ satisfying

$$
\sup \left\{f(x, z, \lambda): z \in[0, r] \backslash D_{f}, \lambda \in[0, r]\right\} \leq \mu_{r}(x)
$$

almost everywhere in $\Omega$.

$\left(h_{5}\right) \lim _{z \rightarrow 0^{+}} f(x, z, 0)=0$ for almost all $x \in \Omega$.

$\left(h_{6}\right)$ For almost every $x \in \Omega$ and every $z \in D_{f}, \lambda \in \mathbb{R}_{0}^{+}, \liminf _{w \notin D_{f}, w \rightarrow z} f(x, w, \lambda)=0$ implies $f(x, z, \lambda)=0$.

$\left(h_{7}\right)$ The problem $L u=\lambda f(x, u, 0)$ in $\Omega, u \in X_{p}(\Omega)$, has no nontrivial solutions for all $\lambda \in] 0,1]$.

Define

$\Sigma_{f}=\left\{(\lambda, u) \in \mathbb{R}_{0}^{+} \times X_{p}(\Omega): L u(x)=f(x, u(x), \lambda)\right.$ almost everywhere in $\left.\Omega\right\}$, where $L$ is given by (1). One clearly has $(0,0) \in \Sigma_{f}$ since, due to $\left(h_{5}\right)$ and $\left(h_{6}\right)$,

$$
f(x, 0,0)=0 \quad \text { for almost every } x \in \Omega \text {. }
$$

We are in a position now to establish the main result of the present paper. It can be regarded as a highly discontinuous version of [23, Theorem 2.12] and [24, Theorem 3.7].

Theorem 2.3. Let $f: \Omega \times \mathbb{R}_{0}^{+} \times \mathbb{R}_{0}^{+} \rightarrow \mathbb{R}_{0}^{+}$be a function fulfilling assumptions $\left(h_{1}\right)$ $\left(h_{7}\right)$. Then there exists an unbounded subcontinuum of the set $\Sigma_{f}$ which contains $(0,0)$.

Proof. Choose $\Omega_{1} \subseteq \Omega$ such that $m\left(\Omega_{1}\right)=0$ and $\left(h_{3}\right)$ - $\left(h_{6}\right)$ hold for all $x \in \Omega \backslash \Omega_{1}$. Because of $\left(h_{1}\right)$ the set $\mathbb{R}_{0}^{+} \backslash D_{f}$ is dense in $\mathbb{R}_{0}^{+}$. So, there exists a countable subset $D$ of $\mathbb{R}_{0}^{+} \backslash D_{f}$ satisfying $\bar{D}=\mathbb{R}_{0}^{+}$. We define, for $(x, z, \lambda) \in \Omega \times \mathbb{R}_{0}^{+} \times \mathbb{R}_{0}^{+}$,

$$
F(x, z, \lambda)= \begin{cases}\bigcap_{k \in \mathbb{N}} c o(\overline{f(x,[z-1 / k, z+1 / k] \cap D, \lambda)}) & \text { if } x \in \Omega \backslash \Omega_{1}, \\ \mathbb{R}_{0}^{+} & \text {otherwise. }\end{cases}
$$

Obviously, $F(x, z, \lambda)$ is convex and closed. Hypothesis $\left(h_{4}\right)$ combined with Cantor's theorem ensures that $F(x, z, \lambda)$ is nonempty and compact whenever $x \in \Omega \backslash \Omega_{1}$. Moreover

$$
F(x, z, \lambda)=\{f(x, z, \lambda)\} \quad \text { for all } x \in \Omega \backslash \Omega_{1}, z \in \mathbb{R}_{0}^{+} \backslash D_{f}, \lambda \in \mathbb{R}_{0}^{+},
$$

as a simple computation shows. 
Let us prove that the multifunction $F: \Omega \times \mathbb{R}_{0}^{+} \times \mathbb{R}_{0}^{+} \rightarrow 2^{\mathbb{R}_{0}^{+}}$has properties $\left(a_{1}\right)-\left(a_{5}\right)$. To this end, pick $z, \lambda \in \mathbb{R}_{0}^{+}$. If $A$ is an open subset of $\mathbb{R}$, then

$$
\begin{aligned}
\{x \in & \left.\Omega \backslash \Omega_{1}: \overline{f(x,[z-1 / k, z+1 / k] \cap D, \lambda)} \cap A \neq \emptyset\right\} \\
& =\left\{x \in \Omega \backslash \Omega_{1}: f(x,[z-1 / k, z+1 / k] \cap D, \lambda) \cap A \neq \emptyset\right\} \\
& =\bigcap_{w \in D,|w-z| \leq 1 / k}\left\{x \in \Omega \backslash \Omega_{1}: f(x, w, \lambda) \in A\right\},
\end{aligned}
$$

namely, by the definition of $D$ and assumption $\left(h_{2}\right)$, the multifunction

$$
x \rightarrow \overline{f(x,[z-1 / k, z+1 / k] \cap D, \lambda)}
$$

is measurable in $\Omega \backslash \Omega_{1}$ for any $k \in \mathbb{N}$. Theorem 9.1 and Corollary 4.2 of [12] lead to the same conclusion for $F$. Hence, condition $\left(a_{1}\right)$ is verified.

Next, fix $x \in \Omega \backslash \Omega_{1}, z, \lambda, y \in \mathbb{R}_{0}^{+}$, and choose two sequences $\left\{\left(z_{h}, \lambda_{h}\right)\right\} \subseteq$ $\mathbb{R}_{0}^{+} \times \mathbb{R}_{0}^{+},\left\{y_{h}\right\} \subseteq \mathbb{R}_{0}^{+}$satisfying $\left(z_{h}, \lambda_{h}\right) \rightarrow(z, \lambda), y_{h} \rightarrow y, y_{h} \in F\left(x, z_{h}, \lambda_{h}\right), h \in \mathbb{N}$. Since to each $k \in \mathbb{N}$ and each $\delta>0$ there corresponds a positive integer $\nu$ such that

$$
\begin{aligned}
y_{h} \in \operatorname{co}\left(\overline{f\left(x,[z-1 / k, z+1 / k] \cap D, \lambda_{h}\right)}\right) \\
\qquad c o\left(\overline{f(x,[z-1 / k, z+1 / k] \cap D,] \lambda-\delta, \lambda+\delta\left[\cap \mathbb{R}_{0}^{+}\right)}\right)
\end{aligned}
$$

for all $h \geq \nu$, we achieve

$$
y \in c o\left(\overline{f(x,[z-1 / k, z+1 / k] \cap D,] \lambda-\delta, \lambda+\delta\left[\cap \mathbb{R}_{0}^{+}\right)}\right) .
$$

Therefore, taking into account hypothesis $\left(h_{3}\right)$,

$$
y \in \operatorname{co}(\overline{f(x,[z-1 / k, z+1 / k] \cap D, \lambda)})
$$

for every $k \in \mathbb{N}$, namely $y \in F(x, z, \lambda)$. We have now showed that the multifunction $(z, \lambda) \rightarrow F(x, z, \lambda)$ has a closed graph. So, by using $\left(h_{4}\right)$, property $\left(a_{2}\right)$ can easily be drawn.

Since $\left(a_{3}\right)$ immediately follows from assumption $\left(h_{4}\right)$, while $\left(a_{4}\right)$ is a simple consequence of $\left(h_{5}\right)$, we only need to verify $\left(a_{5}\right)$. Suppose on the contrary that there exist $\lambda \in] 0,1], u \in X_{p}(\Omega) \backslash\{0\}, \Omega_{2} \subseteq \Omega$ complying with $m\left(\Omega_{2}\right)=0$ and

$$
L u(x) \in \lambda F(x, u(x), 0) \quad \text { for all } x \in \Omega \backslash \Omega_{2} .
$$

Proposition 1.2 yields a set $\Omega_{3} \subseteq u^{-1}\left(D_{f}\right)$ such that

$$
m\left(\Omega_{3}\right)=0 \quad \text { and } \quad L u(x)=0 \quad \text { whenever } x \in u^{-1}\left(D_{f}\right) \backslash \Omega_{3} .
$$

Let $\Omega^{*}=\bigcup_{i=0}^{3} \Omega_{i}$. Evidently, $m\left(\Omega^{*}\right)=0$. Moreover, one has

$$
L u(x)=\lambda f(x, u(x), 0) \quad \text { for every } x \in \Omega \backslash \Omega^{*} .
$$

Indeed, if $x \in \Omega \backslash\left(\Omega^{*} \cup u^{-1}\left(D_{f}\right)\right)$, then $L u(x)=\lambda f(x, u(x), 0)$ because, owing to $(3), L u(x) \in \lambda F(x, u(x), 0)$ and, by $(2), F(x, u(x), 0)=\{f(x, u(x), 0)\}$. If $x \in$ $\left(\Omega \backslash \Omega^{*}\right) \cap u^{-1}\left(D_{f}\right)$, then hypothesis $\left(h_{6}\right)$ together with (3) and (4) lead to $L u(x)=$ $0=\lambda f(x, u(x), 0)$. Consequently, in either case, $L u(x)=\lambda f(x, u(x), 0)$. This means that the problem $L u=\lambda f(x, u, 0)$ in $\Omega, u \in X_{p}(\Omega)$, has nontrivial solutions, against $\left(h_{7}\right)$.

Similar arguments can also be applied to establish the inclusion $\Sigma_{F} \subseteq \Sigma_{f}$. Hence, on account of Theorem 2.1, there exists an unbounded subcontinuum of the set $\Sigma_{f}$ which comprises $(0,0)$, as claimed. 
Remark 2.3. Theorem 2.3 is actually true for any elliptic operator fulfilling the maximum principle and the conclusions of Propositions 1.1 and 1.2. Besides $L$, the linear operators considered in [3], [5], [6], [17], [18] obviously are of this kind.

A direct but significant consequence of Theorem 2.3 is given by the result below. It improves Theorem 4.4 in [17], Theorem 3.3 of [18], and concerning the nonlinearity $f$, exhibits more general assumptions than Theorem 19 in [13] as well as Theorem 5.2 of [7].

Theorem 2.4. Suppose $f$ satisfies hypotheses $\left(h_{1}\right)-\left(h_{4}\right)$, and $\left(h_{6}\right)$. Then the set $\left\{(\lambda, u) \in \mathbb{R}_{0}^{+} \times X_{p}(\Omega): L u(x)=\lambda f(x, u(x), \lambda)\right.$ almost everywhere in $\left.\Omega\right\}$ contains an unbounded subcontinuum emanating from $(0,0)$.

Now, let $\Omega$ be star-shaped relative to some of its points and let $\sigma$ be the critical Sobolev exponent $2 n /(n-2)$. Pick $y^{*} \in \mathbb{R}_{0}^{+}$and choose a bounded real sequence $\left\{y_{h}\right\}$ complying with

$$
\inf _{h \in \mathbb{N}} y_{h}>0, \quad y^{*} \notin\left[\inf _{h \in \mathbb{N}} y_{h}, \sup _{h \in \mathbb{N}} y_{h}\right] .
$$

If $C$ denotes a nonempty closed subset of $\mathbb{R}_{0}^{+}$such that $m(C)=0$ and $0 \in C$, then the set $\mathbb{R}_{0}^{+} \backslash C$ is nonempty and open. So, it has at most countably many connected (open) components $A_{h}, h=1,2, \ldots$ We define, for every $z \in \mathbb{R}_{0}^{+}$,

$$
g(z)= \begin{cases}y_{h} & \text { if } z \in A_{h}, h=1,2, \ldots, \\ y^{*} & \text { if } z \in C\end{cases}
$$

A typical application of Theorem 2.3 is performed in the next result.

Theorem 2.5. Under the above assumptions, there exists an unbounded subcontinuum of the set

$$
\left\{(\lambda, u) \in \mathbb{R}_{0}^{+} \times X_{p}(\Omega): L u(x)=u(x)^{\sigma-1}+\lambda g(u(x)) \text { almost everywhere in } \Omega\right\}
$$

which comprises $(0,0)$.

Proof. Define $f(x, z, \lambda)=z^{\sigma-1}+\lambda g(z),(x, z, \lambda) \in \Omega \times \mathbb{R}_{0}^{+} \times \mathbb{R}_{0}^{+}$. Clearly, owing to Theorem 2.3, the conclusion is achieved once we verify that the function $f$ has properties $\left(h_{1}\right)-\left(h_{7}\right)$. The first of them is fulfilled because $D_{f}=C$ and, by hypothesis, $m(C)=0$. Elementary arguments, mainly based upon the boundedness of the sequence $\left\{y_{h}\right\}$ and the inequality $\inf _{h \in \mathbb{N}} y_{h}>0$, yield the subsequent five. Finally, taking into account the classical example by Pohožaev [21, p. 1410], we see that condition $\left(h_{7}\right)$ is true too.

\section{REFERENCES}

[1] R.A. Adams: Sobolev Spaces, Academic Press, New York, 1975. MR 56:9247

[2] H. Amann, Fixed point equations and nonlinear eigenvalue problems in ordered Banach spaces, SIAM Rev. 18 (1976), 620-709. MR 54:3519

[3] A. Ambrosetti, M. Calahorrano, and F. Dobarro, Global branching for discontinuous problems, Comment. Math. Univ. Carolin. 31 (1990), 213-222. MR 91m:35229

[4] J. Appell, E. De Pascale, H.T. Nguyêñ, and P.P. Zabreǐko, Multi-valued superpositions, Dissertationes Math. 345 (1995), 1-97. MR 96h:47061

[5] M. Badiale and G. Tarantello, Existence and multiplicity results for elliptic problems with critical growth and discontinuous nonlinearities, Nonlinear Anal., to appear.

[6] G. Bonanno and S.A. Marano, Positive solutions of elliptic equations with discontinuous nonlinearities, Topol. Methods Nonlinear Anal. 8 (1996), to appear.

[7] K.C. Chang, Free boundary problems and the set-valued mappings, J. Differential Equations 49 (1983), 1-28. MR 84h:35172 
[8] F. Chiarenza, M. Frasca, and P. Longo, $W^{2, p}$-solvability of the Dirichlet problem for nondivergence elliptic equations with VMO coefficients, Trans. Amer. Math. Soc. 336 (1993), 841-853. MR 93f:35232

[9] E. De Giorgi, G. Buttazzo, and G. Dal Maso, On the lower semicontinuity of certain integral functionals, Atti Accad. Naz. Lincei Cl. Sci. Fis. Mat. Natur. Rend. Lincei (8) Mat. Appl. 74 (1983), 274-282. MR 87a:49019

[10] D. Gilbarg and N.S. Trudinger, Elliptic Partial Differential Equations of Second Order, 2nd edn., Springer-Verlag, Berlin, 1983. MR 86c:35035

[11] S. Heikkilä and V. Lakshmikantham, Monotone Iterative Techniques for Discontinuous Nonlinear Differential Equations, Marcel Dekker, New York, 1994. MR 95d:34002

[12] C.J. Himmelberg, Measurable relations, Fund. Math. 87 (1975), 53-72. MR 51:3384

[13] H.J. Kuiper, On positive solutions of nonlinear elliptic eigenvalue problems, Rend. Circ. Mat. Palermo (2) 20 (1971), 113-138. MR 48:6680

[14] D. Lupo, A bifurcation result for a Dirichlet problem with discontinuous nonlinearity, Rend. Circ. Mat. Palermo (2) 38 (1989), 305-318. MR 91a:35026

[15] S.A. Marano, Elliptic boundary-value problems with discontinuous nonlinearities, Set-Valued Anal. 3 (1995), 167-180. MR 96j:35079

[16] S.A. Marano, Implicit elliptic boundary-value problems with discontinuous nonlinearities, Set-Valued Anal. 4 (1996), 287-300. CMP 97:02

[17] I. Massabò and C.A. Stuart, Elliptic eigenvalue problems with discontinuous nonlinearities, J. Math. Anal. Appl. 66 (1978), 261-281. MR 80f:35102

[18] I. Massabò, Positive eigenvalues for elliptic equations with discontinuous nonlinearities, Boll. Un. Mat. Ital. B (5) 15 (1978), 814-827. MR 80a:35051

[19] D.K. Palagachev, Quasilinear elliptic equations with VMO coefficients, Trans. Amer. Math. Soc. 347 (1995), 2481-2493. MR 95k:35083

[20] D.K. Palagachev, private communication.

[21] S.I. Pohožaev, Eigenfunctions of the equation $\Delta u+\lambda f(u)=0$, Soviet Math. Dokl. 6 (1965), 1408-1411.

[22] T. Pruszko, Topological degree methods in multi-valued boundary value problems, Nonlinear Anal. 5 (1981), 959-973. MR 83d:34034

[23] P.H. Rabinowitz, Some global results for nonlinear eigenvalue problems, J. Funct. Anal. 7 (1971), 487-513. MR 46:745

[24] P.H. Rabinowitz, A global theorem for nonlinear eigenvalue problems and applications, in E. Zarantonello (ed.), Contributions to Nonlinear Functional Analysis, Academic Press, New York, 1971, pp. 11-36. MR 52:11681

Department of Mathematics, University of Catania, Viale A. Doria 6, 95125 Catania, ITALY

E-mail address: marano@dipmat.unict.it 